\title{
On developed superfluid turbulence
}

\author{
G.E. Volovik \\ Low Temperature Laboratory, Helsinki University of Technology, P.O.Box 2200, \\ FIN-02015 HUT, Finland \\ L.D. Landau Institute for Theoretical Physics, Kosygin Str. 2, 117940 Moscow, \\ Russia
}

Superfluid turbulence is governed by two dimensionless parameters. One of them is the intrinsic parameter $q$ which characterizes the relative value of the friction force acting on a vortex with respect to the non-dissipative forces. The inverse parameter $q^{-1}$ plays the same role as the Reynolds number $\operatorname{Re}=U R / \nu$ in classical hydrodynamics. It marks the transition between the "laminar" and turbulent regimes of vortex dynamics. The developed turbulence, described by a Kolmogorov cascade, occurs when Re $\gg 1$ in classical hydrodynamics. In superfluids, the developed turbulence occurs at $q \ll 1$. Another parameter of superfluid turbulence is the superfluid Reynolds number $\operatorname{Re}_{s}=U R / \kappa$, which contains the circulation quantum $\kappa$ characterizing quantized vorticity in superfluids. The two parameters $q$ and $\operatorname{Re}_{s}$ control the crossover or transition between two classes of superfluid turbulence: (i) the classical regime, where the Kolmogorov cascade (probably modified by the non-canonical dissipation due to mutual friction) is effective, vortices are locally polarized, and the quantization of vorticity is not important; and (ii) the Vinen quantum turbulence where the properties are determined by the quantization of vorticity. The phase diagram of these dynamical vortex states is suggested.

PACS numbers: 43.37.+q, 47.32.Cc, 67.40. Vs, 67.57.Fg

\section{Introduction}

Recent experiments in ${ }^{3} \mathrm{He}-\mathrm{B}^{1}$ demonstrated that the fate of few vortices injected into a rapidly moving superfluid depends on dimensionless intrinsic temperature dependent parameter $q$, rather than on the flow velocity. At 


\section{G.E. Volovik}

$q$ of order unity a rather sharp transition is observed between the laminar evolution of the injected vortices and the emerging turbulent many-vortex state of the whole superfluid. This added a new twist to the general theory of turbulence in superfluids developed by Vinen ${ }^{2,3}$ and others. Attempts to modify the theory in order to incorporate the new phenomenon, have been made in Refs. ${ }^{5}$ and. ${ }^{6}$ Here we follow the latter work ${ }^{6}$ where we utilized the coarse-grained hydrodynamic equation for the dynamics of the superfluid with distributed vortices. In this equation the parameter $q$ characterizes the friction force between the superfluid and normal components of the liquid, which is mediated by quantized vortices. According to this equation, turbulence develops only if the friction is relatively small compared to the inertial term, i.e. when $q$ is below unity. We also argued that the developed turbulence must occur at $q \ll 1$, and suggested that in this region, where the inertial term is dominating, there are at least two possible states of turbulence. One of them corresponds to the state discussed by Vinen, where the microscopic nature of quantized vortices is essential. While in the other state, turbulence does not depend on the circulation quantum, and thus the information on the underlying microscopic physics of quantum liquid is lost. Turbulence in this state becomes similar to turbulence in classical liquids, and it is also described by the Richardson cascade at least at the initial stage of the development. However, as distinct from the classical liquids, the final state of turbulence is determined not by viscosity but by the mutual friction parameter $q$.

These differences from the classical turbulence arise because the hydrodynamics of superfluid liquid exhibits new features with respect to conventional classical hydrodynamics, which become important when turbulence in superfluids is considered:

(i) The superfluid liquid consists of mutually penetrating components - the viscous normal component and one or several frictionless superfluid components. That is why different types of turbulent motion are possible depending on whether the normal and superfluid components move together or separately. Here we are interested in the most simple case when the dynamics of the normal component can be neglected. This occurs, for example, in the superfluid phases of ${ }^{3} \mathrm{He}$ where the normal component is so viscous that it is practically clamped to the container walls. The role of the normal component in this case is to provide the preferred heat-bath reference frame, where the normal component and thus the heat bath are at rest. The dissipation takes place when vortices move with respect to this reference frame. The turbulence in the superfluid component with the normal component at rest is here referred to as superfluid turbulence. We also assume that there is only a single superfluid component. For ${ }^{3} \mathrm{He}-\mathrm{B}$ this means that we ignore the 


\section{On developed superfluid turbulence}

spin degrees of freedom, assuming that all three superfluid spin components move together.

(ii) The important feature of superfluid turbulence is that the vorticity of the superfluid component is quantized in terms of the elementary circulation quantum $\kappa$ (in ${ }^{3} \mathrm{He}-\mathrm{B} \kappa=\pi \hbar / m$, where $m$ is the mass of ${ }^{3} \mathrm{He}$ atom). So, superfluid turbulence is the chaotic motion of well determined and well separated vortex filaments. ${ }^{3}$ Using this as starting point we can simulate the main ingredients of classical turbulence - the chaotic dynamics of the vortex degrees of freedom of the liquid. However, to make the analogy useful one must choose the regime where the microscopic nature of the superfluid - the circulation quantum containing the Planck constant $\hbar$ and the mass of the atom $m$ - is not involved.

(iii) The most important distinction from the classical hydrodynamics is that the dissipation in vortex motion is not due to the viscosity term $\nu \nabla^{2} \mathbf{v}$ in the Navier-Stokes equation. The superfluid component does not exhibit viscosity, instead the dissipation occurs due to the friction force acting on the superfluid vortex when it moves with respect to the heat bath (the normal component). The force acting on a single vortex is proportional to the velocity of the vortex in the heat-bath frame. In the coarse-grained hydrodynamics of the superfluid with the distributed vorticity, this leads to the force between the superfluid and normal components, which is proportional to their relative velocity $\mathbf{v}_{\mathrm{s}}-\mathbf{v}_{\mathrm{n}}$, and to the vortex density $\left|\nabla \times \mathbf{v}_{\mathrm{s}}\right|$. As a result the dissipative term in the hydrodynamic equation is non-linear, and its structure reminds that of the non-linear inertial term. The relative magnitude of the two non-linear terms, the friction and inertial ones, is given by the dimensionless parameter $q$. Thus the quantity $1 / q$ plays the role of the Reynolds number. This is an internal parameter of the liquid, as distinct form the Reynolds number in conventional liquids where it depends on the flow velocity and dimension of the system. When $q \ll 1$, the inertial term is dominating, and this corresponds to a big Reynolds number in classical liquids. In this regime one can expect, that the turbulent state obeys the Richardson energy cascade, governed by the inertial term. We discuss here whether the Kolmogorov scaling law survives the non-canonical dissipation in superfluids, i.e. whether or not the scaling is modified due to the nonlinear dissipative term.

\section{Coarse-grained hydrodynamic equation}

The coarse-grained hydrodynamic equation is obtained from the Euler equation for the superfluid velocity $\mathbf{v} \equiv \mathbf{v}_{\mathbf{s}}$ after averaging over the vortex lines (see Refs. ${ }^{7}$ and review $^{8}$ ). Instead of the Navier-Stokes equation with 


\section{G.E. Volovik}

the $\nu \nabla^{2} \mathbf{v}$ term one has

$$
\begin{array}{r}
\frac{\partial \mathbf{v}}{\partial t}+\nabla \mu=\mathbf{v} \times \vec{\omega}- \\
-\alpha^{\prime}\left(\mathbf{v}-\mathbf{v}_{\mathrm{n}}\right) \times \vec{\omega}+\alpha \hat{\omega} \times\left(\vec{\omega} \times\left(\mathbf{v}-\mathbf{v}_{\mathrm{n}}\right)\right) .
\end{array}
$$

Here $\mathbf{v}_{\mathbf{n}}$ is the velocity of the normal component; $\vec{\omega}=\nabla \times \mathbf{v}$ is the superfluid vorticity; $\hat{\omega}=\vec{\omega} / \omega$; and the dimensionless parameters $\alpha^{\prime}$ and $\alpha$ come from the reactive and dissipative forces acting on a vortex when it moves with respect to the normal component. These parameters are very similar to the Hall and conventional resistivities, $\rho_{x y}$ and $\rho_{x x}$, in the Hall effect. For vortices in fermionic systems (superfluid ${ }^{3} \mathrm{He}$ and superconductors) they were calculated by Kopnin, ${ }^{9}$ and measured in ${ }^{3} \mathrm{He}-\mathrm{B}$ over a broad temperature range by Bevan et. al. ${ }^{10}$ (see also, ${ }^{11}$ where these parameters are discussed in terms of the chiral anomaly). We omitted the higher order terms, including the term discussed by Hall ${ }^{7}$ which contains the bending energy of vortex lines (the condition for that is discussed in Section 6 where another dimensionless parameter will be introduced - the superfluid Reynolds number $\operatorname{Re}_{s}=U R / \kappa$ which contains the circulation quantum $\kappa$ ).

The terms in expression (1) are invariant with respect to the transformation $\mathbf{v} \rightarrow \mathbf{v}(\mathbf{r}-\mathbf{u} t)+\mathbf{u}$ as in classical hydrodynamics. However, the terms in expression (2) are not invariant under this transformation, since there is the preferred reference frame in which the normal component is at rest. They are invariant under the full Galilean transformation when the normal component is also involved: $\mathbf{v} \rightarrow \mathbf{v}(\mathbf{r}-\mathbf{u} t)+\mathbf{u}$ and $\mathbf{v}_{\mathrm{n}} \rightarrow \mathbf{v}_{\mathrm{n}}+\mathbf{u}$.

The experiments in Ref. ${ }^{1}$ were made in a rotating cryostat, where the normal component exhibits solid body rotation, $\mathbf{v}_{\mathrm{n}}=\boldsymbol{\Omega} \times \mathbf{r}$. However, here we study the local properties of turbulence and avoid this complication, assuming that $\mathbf{v}_{\mathbf{n}}$ is uniform. Actually this means that we consider the case of strong turbulence where the vortex density essentially exceeds the equilibrium vortex density in the rotating container: $\omega \gg \Omega$. We shall work in the frame comoving with the normal component, where $\mathbf{v}_{\mathrm{n}}=0$, but we must remember that this frame is unique. In this frame the equation for superfluid hydrodynamics is simplified:

$$
\frac{\partial \mathbf{v}}{\partial t}+\nabla \mu=\left(1-\alpha^{\prime}\right) \mathbf{v} \times \vec{\omega}+\alpha \hat{\omega} \times(\vec{\omega} \times \mathbf{v}) .
$$

After rescaling the time, $\tilde{t}=\left(1-\alpha^{\prime}\right) t$, one obtains an equation

$$
\frac{\partial \mathbf{v}}{\partial \tilde{t}}+\nabla \tilde{\mu}=\mathbf{v} \times \vec{\omega}+q \hat{\omega} \times(\vec{\omega} \times \mathbf{v})
$$

which depends on a single parameter $q$ :

$$
q=\frac{\alpha}{1-\alpha^{\prime}}
$$




\section{On developed superfluid turbulence}

Now in Eq.(4) the first three terms together are the same as the inertial terms in classical hydrodynamics. They satisfy the modified Galilean invariance:

$$
\mathbf{v}(\tilde{t}, \mathbf{r}) \rightarrow \mathbf{v}(\tilde{t}, \mathbf{r}-\mathbf{u} \tilde{t})+\mathbf{u}
$$

In fact the transformation above changes the chemical potential, but this does not influence the vortex degrees of freedom which are important for the phenomenon of turbulence. In contrast, the dissipative last term with the factor $q$ in Eq.(4) is not invariant under this transformation. This is in contrast to the conventional liquid where the whole Navier-Stokes equation, which contains the kinematic viscosity

$$
\frac{\partial \mathbf{v}}{\partial t}+\nabla \mu=\mathbf{v} \times \vec{\omega}+\nu \nabla^{2} \mathbf{v},
$$

is Galilean invariant, and where there is no preferred reference frame.

This difference between the dissipative last terms in Eqs. (7) and (4) is very important:

(1) The role of the Reynolds number, which characterizes the ratio of the inertial and dissipative terms in the hydrodynamic equations, is played by the intrinsic parameter $1 / q$ in superfluid turbulence. This parameter does not depend on the characteristic velocity $U$ and size $R$ of the large-scale flow as distinct from the conventional Reynolds number $\operatorname{Re}=R U / \nu$ in classical viscous hydrodynamics. That is why the turbulent regime occurs only at $1 / q>1$ even if vortices are injected to the superfluid which moves with large velocity $U$. This rather unexpected result was obtained in experiments with superfluid ${ }^{3} \mathrm{He}-\mathrm{B} .{ }^{1}$

(2) In conventional turbulence the large-scale velocity $U$ is always understood as the largest characteristic velocity difference in the inhomogeneous flow of the classical liquid. ${ }^{12}$ In the two-fluid system the velocity $U$ is the large-scale velocity of the superfluid component with respect to the normal component, and this velocity (the so-called counterflow velocity) can be completely homogeneous (however, for the intermediate turbulent state obtained in the ${ }^{3} \mathrm{He}-\mathrm{B}$ experiments in rotating vessel, the large-scale velocity field is inhomogeneous and we use $U \sim \Omega R$, with $\Omega$ being the rotation velocity and $R$ - the radius of the container).

(3) As distinct from the classical hydrodynamics, the energy dissipation is produced by the non-linear mutual friction ( $\mathrm{mf}$ ) term - the last term in Eq.(4):

$$
\epsilon_{\mathrm{mf}}=-\dot{E}=-\left\langle\mathbf{v} \cdot \frac{\partial \mathbf{v}}{\partial \tilde{t}}\right\rangle=-q\langle\mathbf{v} \cdot(\hat{\omega} \times(\vec{\omega} \times \mathbf{v}))\rangle \sim q \omega v^{2} .
$$

This reflects the fact that the mutual friction depends on the velocity of the superfluid component with respect to the normal one, i.e. on the absolute 


\section{G.E. Volovik}

value of the velocity $v$ in the frame of the container. Thus for a given value of vorticity $\omega$ the largest dissipation occurs at the large-scale velocity $U$. On the other hand, according to the Kolmogorov scaling, the maximum vorticity $\omega$ is concentrated at the smallest scale. This gives the nontrivial dependence of the dissipation on scales: dissipation is not concentrated at the smallest scale only.

(4) The onset of the superfluid turbulence was studied by Kopnin in Ref. $^{5}$ His Vinen-type model demonstrated that the initial avalanche-like multiplication of vortices leading to turbulence occurs when $q$ drops below unity, which is in agreement with experiment. ${ }^{1}$ The existence of two regimes in the initial development of vorticity is also supported by earlier simulations by Schwarz who noted that when $\alpha$ (or $q$ ) is reduced a crossover from a regime of isolated phase slips to a phase-slips cascade and then to the fully developed vortex turbulence occurs. ${ }^{13}$

(5) One can expect that the well developed turbulence occurs when $q \ll 1$, and here we shall discuss this extreme limit. In ${ }^{3} \mathrm{He}-\mathrm{B}$ the condition $q \ll 1$ is realized at temperatures well below $0.6 T_{c} \cdot{ }^{1}$ However, we do not consider very low temperatures where instead of the mutual friction the other mechanisms of dissipation take place such as excitation of Kelvin waves ${ }^{14}$ and vortex reconnection. ${ }^{15}$ The latter leads to formation of cusps and kinks on the vortex filaments whose fast dynamics creates a burst of different types of excitations in quantum liquids: phonons, rotons, Kelvin waves and fermionic quasiparticles. A similar burst of gravitational waves from cusps and kinks on cosmic strings was theoretically investigated by the cosmological community (see e.g. ${ }^{16}$ ), and the obtained results are very important for the superfluid turbulence at a very low temperature.

(6) We expect that even at $q \ll 1$, two different states of turbulence are possible, with the crossover (or transition) between them being determined by $q$ and by another dimensionless parameter $\operatorname{Re}_{s}=U R / \kappa$, where $\kappa$ is the circulation around the quantum vortex. The coarse-grained hydrodynamic equation (4) is in fact valid only in the limit $\operatorname{Re}_{s} \gg 1$, since the latter means that the characteristic circulation of the velocity $\Gamma=U R$ of the large-scale flow substantially exceeds the circulation quantum $\kappa$, and thus there are many vortices in the turbulent flow. When $\operatorname{Re}_{s}$ is reduced the quantum nature of vortices becomes more pronounced. At some (still big) value of $\operatorname{Re}_{s}$ we proceed from the type of superfluid turbulence which resembles the classical turbulence and is probably described by the (modified) Kolmogorov cascade, to the quantum regime which is probably described by the Vinen equations for the average vortex dynamics. ${ }^{2}$ 


\section{On developed superfluid turbulence}

\section{Kolmogorov cascade}

Let us first consider the possibility of a Kolmogorov-Richardson cascade in superfluid turbulence. Let us start with the analysis based on the dimensionalities and on the idea of the energy cascade. In the next Section we shall use a more detailed analysis based on model dynamical equations for the energy spectrum and on dispersion of dissipation over the scales.

In classical turbulence, a large Reynolds number $\operatorname{Re}=U R / \nu \gg 1$ leads to well separated length scales or wave numbers. As a result the KolmogorovRichardson cascade takes place in which the energy flows from small wave numbers $k_{\text {min }} \sim 1 / R$ (large rings of size $R$ of the container) to high wave number $k_{0}=1 / r_{0}$ where the dissipation occurs. In the same manner in our case of superfluid turbulence the necessary condition for a Kolmogorov cascade is a large ratio of the inertial and dissipative terms in Eq.(4), i.e. $1 / q \gg 1$.

In the Kolmogorov-Richardson cascade, at arbitrary length scale $r$ the energy transfer rate to the smaller scale, say $r / 2$, is $v_{r}^{2} / t_{r}$, where $v_{r}^{2}$ is the kinetic energy at this scale, and $t_{r}=r / v_{r}$ is the characteristic time (turnover time). If there are no appreciable losses of energy in the intermediate scales, the energy transfer from one scale to the next must be the same for all scales. As a result one has

$$
\epsilon=\frac{v_{r}^{2}}{t_{r}}=\frac{v_{r}^{3}}{r}=\text { constant }=\frac{U^{3}}{R} .
$$

From this equation it follows that

$$
v_{r}=\epsilon^{1 / 3} r^{1 / 3} .
$$

This certainly occurs in classical liquids, and there is some indication that this might take place in superfluids too. ${ }^{20}$ However, in superfluids the dissipation mechanism is different, which can change the overall picture: even if initially the energy flows to the smaller scale according to the Richardsonin, this does not mean that in the final steady state the Kolmogorov scaling will be established.

In classical liquids, the energy dissipation due to viscosity is $\epsilon_{\mathrm{visc}} \sim$ $\nu\left(\nabla_{i} \mathbf{v}\right)^{2}$. It is concentrated at the smallest scale $r_{0}$, which is found from the energy balance $\epsilon=\epsilon_{\mathrm{visc}} \sim \nu v_{0}^{2} / r_{0}^{2}=\nu \epsilon^{2 / 3} r_{0}^{-4 / 3}$. This gives

$$
r_{0}=1 / k_{0} \sim R \operatorname{Re}^{-3 / 4} .
$$

In superfluid turbulence, the situation is more complicated. According to Eq.(8), in superfluids the dissipation is not a linear function of the velocity. In the non-linear theory, as follows from the Fourier analysis, contributions 


\section{G.E. Volovik}

from different scales are not independent. Since the vorticity $\omega_{r}=v_{r} / r$ is concentrated at the smallest scale, while the velocity - at the largest scales, one obtains that the overall dissipation due to mutual friction is $\epsilon_{\mathrm{mf}} \sim q \omega_{0} U^{2}$. Let us make a natural assumption that the Kolmogorov cascade stops when the overall dissipation becomes comparable to the energy transfer from scale to scale $\epsilon$ in Eq.(9)

$$
\epsilon=\epsilon_{\mathrm{mf}} \sim q \omega_{0} U^{2} \sim q U^{2} \frac{v_{0}}{r_{0}}=q U^{2} \epsilon^{1 / 3} r_{0}^{-2 / 3} \sim \frac{U^{3}}{R} .
$$

Then, from the above equation one obtains the smallest length scale in the superfluid turbulence:

$$
r_{0}=1 / k_{0} \sim q^{3 / 2} R,
$$

which depends on the internal parameter $q$ instead of the Reynolds number Re in Eq.(11) for classical liquids. The associated characteristic velocity $v_{0}$ and vorticity $\omega_{0}$ at this scale are

$$
v_{0} \sim q^{1 / 2} U, \omega_{0}=k_{0} v_{0} \sim \frac{U}{q R} .
$$

This consideration is valid when $r_{0} \ll R$ and $v_{0} \ll U$, which means that $1 / q \gg 1$ is the condition for the Kolmogorov cascade. In classical liquids the corresponding condition for well developed turbulence is Re $\gg 1$. In both cases these conditions ensure that the kinetic terms in the hydrodynamic equations are much larger than the dissipative terms in a large enough inertial range. In the same manner as in classical liquids the condition for the stability of the turbulent flow is $\operatorname{Re}>1$, one may suggest that the condition for the stability of the discussed turbulent flow is $1 / q>1$. This is supported by observations in ${ }^{3} \mathrm{He}-\mathrm{B}$ where it was demonstrated that at high velocity $U$ but at $q>1$, turbulence is not developed even after vortices were introduced into the flow. ${ }^{1}$

In the Kolmogorov cascade both in classical liquids and superfluids the kinetic energy is concentrated at large scales comparable to the container size:

$$
E=\int_{r_{0}}^{R} \frac{d r}{r} v_{r}^{2}=\int_{r_{0}}^{R} \frac{d r}{r}(\epsilon r)^{2 / 3}=(\epsilon R)^{2 / 3}=U^{2} .
$$

The dispersion of the turbulent energy in the momentum space is also the same as in classical liquid

$$
\begin{array}{r}
E=\int_{r_{0}}^{R} \frac{d r}{r} v_{r}^{2}=\int_{k_{0}}^{1 / R} \frac{d k}{k} v_{k}^{2}=\int_{k_{0}}^{1 / R} \frac{d k}{k} \frac{\epsilon^{2 / 3}}{k^{2 / 3}}=\int_{k_{0}}^{1 / R} d k E(k), \\
E(k)=v_{k}^{2} / k=\epsilon^{2 / 3} k^{-5 / 3} .
\end{array}
$$




\section{On developed superfluid turbulence}

However, the dispersion of dissipation caused by the mutual friction is different from that in viscous classical liquids, where the main dissipation occurs at the smallest scale. This can change the whole pattern of the turbulent steady state.

\section{Dispersion of dissipation}

In superfluids the direct transfer of the kinetic energy to the normal component due to the mutual friction occurs at all scales. Let us consider the dispersion $\epsilon_{r}^{\mathrm{mf}}$ of this dissipation as a function of the length scale $r$. In the nonlinear term $q \omega v^{2}$ the given scale $r$ contributes through the velocity field $\mathbf{v}$ and the vorticity field $|\omega|$. The most important contributions are from the fluctuating vorticity $\omega_{r}$ and the fluctuating velocity $v_{r}$ :

$$
\epsilon_{r}^{\mathrm{mf}}=\epsilon_{r}^{(1)}+\epsilon_{r}^{(2)}=q\left\langle\frac{v^{2}}{\omega}\right\rangle \omega_{r}^{2}+q\langle\omega\rangle v_{r}^{2} .
$$

The term $\epsilon_{r}^{(1)}$ comes from the dissipation experienced by the fluctuating vorticity $\omega_{r}$ at scale $r$. Assuming the Kolmogorov scaling one obtains the following estimation of this term

$$
\epsilon_{r}^{(1)}=q\left\langle\frac{v^{2}}{\omega}\right\rangle \omega_{r}^{2}=q \frac{\left\langle v^{2}\right\rangle}{\langle\omega\rangle} \omega_{r}^{2}=\frac{q U^{2}}{\omega_{0}} \omega_{r}^{2}=\epsilon\left(r_{0} / r\right)^{4 / 3},
$$

Here as before $\epsilon=U^{3} / R$ is the energy flux from scale to scale in the inertial range. We take into account that the vorticity moves with respect to the normal component with the characteristic velocity $U$ and thus experiences the corresponding mutual friction. The Eq.(18) corresponds to the effective turbulent viscosity: $\epsilon_{r}^{(1)}=\nu_{\text {eff }} \omega_{r}^{2}$ with $\nu_{e f f}=q U^{2} / \omega_{0}$. As in the classical liquids this dissipation is peaked at the smallest scale where the vorticity is concentrated.

The second term in Eq.(17) is the contribution of the velocity fluctuations to the friction experienced by the average vorticity (see Eq.(16) in Ref. $^{4}$ ). Under the same assumption of the Kolmogorov scaling, the second term has the following magnitude:

$$
\epsilon_{r}^{(2)}=q \omega_{0} v_{r}^{2}=\epsilon(r / R)^{2 / 3} .
$$

Though the average vorticity is concentrated at the smallest scale, $\langle\omega\rangle=\omega_{0}$, the term $\epsilon_{r}^{(2)}$ as a function of $r$ is peaked at largest scale $r=R$ where the velocity and kinetic energy are concentrated. This term which removes energy at large scales was also considered in classical liquids (see e.g. Eq.(5) in Ref. ${ }^{17}$ with $\left.\alpha=q \omega_{0}\right)$. 


\section{G.E. Volovik}

The other possible terms in the dissipation are smaller than these two if $q \ll 1$. For example, one can add the term $q v_{r}^{2} \omega_{r}$. In the Kolmogorov cascade it does not depend on the scale $r$, and is always much smaller than the sum of the two main terms: $q \omega_{r} v_{r}^{2}=q \epsilon \ll q^{2 / 3} \epsilon=\min _{r}\left(\epsilon_{r}^{(1)}+\epsilon_{r}^{(2)}\right)$. Thus at $q \ll 1$ we have two peaks where the dissipation is concentrated. These two peaks at two extreme scales, $r_{0}$ and $R$, have equal magnitude if the Kolmogorov scaling is obeyed:

$$
\epsilon_{r=r_{0}}^{(1)} \sim \epsilon_{r=R}^{(2)} \sim \epsilon=U^{3} / R
$$

Let us now assume that dissipation which occurs at a given scale $r$ is on the order of the losses of the kinetic energy at the same scale, i.e. $\partial_{t} E_{r} \sim-\epsilon_{r}$. Since the dissipation is nonlinear, this is not the fact; nevetheless, this is a rather natural assumption. If one accepts this at least for the two scales, $R$ and $r_{0}$, then using the double-peak structure of the dispersion of the dissipation one comes to the following scenario of the cascade. The part of the initial kinetic energy of the superflow at large scale $r=R$ is directly transferred to the normal component of the liquid due to the mutual friction in Eq.(19). The remaining comparable part of the flow energy experiences the Kolomogorov-Richardson cascade to the smaller scales until the next dissipation peak due to Eq.(18) is reached at $r=r_{0}$.

\section{Possible modifications of the Kolmogorov cascade due to dispersion of dissipation}

If our assumption is correct, the above scenario with two peaks of dissipation does not change the Kolmogorov scaling discussed in Sec. 3, though some part of the kinetic energy is dissipated already at large scale. However, in principle, the dispersion of the dissipation can modify the energy cascade and the scaling law. Let us consider this using the following model of dissipation, which probably is not very realistic but allows us to find the modified scaling law within the model. Let us suppose that the two peaks overlap so that instead of the double-peak structure, the dissipation due to the direct transfer of the energy to the normal component is equally distributed over the scales:

$$
\epsilon_{r}^{\mathrm{mf}}=\epsilon^{\mathrm{mf}} \sim q \omega_{0} U^{2}
$$

and consider, whether the picture of the Kolmogorov-Richardson cascade survives or not under this new input. And if yes, how this modifies the Kolmogorov spectrum. Due to the direct losses of energies at each scale the energy transfer from scale to scale $\epsilon_{r}^{\text {cascade }}$ decreases with decreasing scale, 


\section{On developed superfluid turbulence}

so that the Eq.(9) does not hold any more:

$$
\epsilon_{r}^{\text {cascade }}=\frac{v_{r}^{2}}{t_{r}}=\frac{v_{r}^{3}}{r} \neq \frac{U^{3}}{R} \equiv \epsilon
$$

Instead we must write an equation for the $r$-dependent $\epsilon_{r}^{\text {cascade }}$ in the steady state, which takes into account that the energy flux from the scale $r$ to the neighbouring scale $r / 2$ splits into the direct energy transfer from the scale $r / 2$ to the normal component and the energy flux to the next scale: $\epsilon_{r}^{\text {cascade }}=\epsilon_{r / 2}^{\text {cascade }}+\epsilon_{\mathrm{mf}}$. Thus the difference between the energy fluxes at neighbouring scales is balanced by the direct energy losses at each step in Eq.(21) and one obtains the following equation for $\epsilon_{r}^{\text {cascade: }}$

$$
r \partial_{r} \epsilon_{r}^{\text {cascade }}=\epsilon_{\mathrm{mf}}, \quad \text { or } \quad r \partial_{r}\left(\frac{v_{r}^{3}}{r}\right)=\epsilon_{\mathrm{mf}} .
$$

In terms of wave numbers $k=1 / r$ and taking into account the time dependence, the energy balance in Eq.(23) can be written as

$$
\partial_{t}\left(v_{k}^{2}\right)+k \partial_{k}\left(k v_{k}^{3}\right)=-\epsilon^{\mathrm{mf}}, \text { or } \quad \partial_{t} E(k)+\partial_{k}\left(k^{5 / 2} E^{3 / 2}\right)=-\frac{\epsilon_{\mathrm{mf}}}{k} .
$$

where as before the energy $E(k)=v_{k}^{2} / k$.

We can also use the more sophisticated equations of Leith type describing the diffusion of energy in the $k$-space, which have been considered for turbulent cascades in conventional liquids. ${ }^{18}$ For example, in a recent publication ${ }^{19}$ the following diffusion equation was used:

$$
\partial_{t} E=\frac{1}{8} \partial_{k}\left(k^{11 / 2} E^{1 / 2} \partial_{k}\left(E / k^{2}\right)\right)+f-\nu k^{2} E .
$$

Here $f$ is an external source of energy. In superfluids, instead of the last (viscous) term, there is a sink of energy caused by the direct transfer of energy to the normal component by mutual friction. In our model with constant dispersion of dissipation one has:

$$
\partial_{t} E=\frac{1}{8} \partial_{k}\left(k^{11 / 2} E^{1 / 2} \partial_{k}\left(E / k^{2}\right)\right)+f-\frac{1}{k} \epsilon_{\mathrm{mf}} .
$$

For this model the steady-state solutions of equations (24) and (26) are the same (assuming that the source term $f(k)=0$ at $k>1 / R$ ):

$$
E(k)=C\left(\epsilon_{\mathrm{mf}}\right)^{2 / 3} k^{-5 / 3} \ln ^{2 / 3} \frac{k_{0}}{k} .
$$

They differ only by the prefactor $C$ which is $C=(24 / 11)^{2 / 3}$ for Eq.(26), and $C=1$ for Eq.(24). 


\section{G.E. Volovik}

Eq.(27) demonstrates the main difference in the energy spectrum for the conventional and modified Kolmogorov cascades. In both cases the energy spectrum can be represented as

$$
E(k)=C \epsilon^{2 / 3} k^{-5 / 3} F\left(k / k_{0}\right) .
$$

But in conventional turbulence one has $F\left(k \ll k_{0}\right)=1$, while in the modified turbulence the function $F\left(k / k_{0}\right)$ logarithmically diverges at $k_{0} \gg k$.

From Eq.(27) it follows that the velocity

$$
v_{k}=(k E)^{1 / 2} \sim\left(\epsilon_{\mathrm{mf}}\right)^{1 / 3} k^{-1 / 3} \ln ^{1 / 3} \frac{k_{0}}{k}
$$

monotonically decreases with $k$ and approaches zero at $k \rightarrow k_{0}$. On the other hand the vorticity

$$
\omega_{k}=k v_{k} \sim\left(\epsilon_{\mathrm{mf}}\right)^{1 / 3} k^{2 / 3} \ln ^{1 / 3} \frac{k_{0}}{k}
$$

first increases, then reaches its maximum

$$
\omega_{\max } \sim\left(\epsilon_{\mathrm{mf}}\right)^{1 / 3} k_{0}^{2 / 3}
$$

at $k_{\max }=k_{0} / \sqrt{e}$ and finally almost abrubtly goes to zero within the same scale $k_{0}$.

The kinetic energy $E(k)$ decreases first as $k^{-5 / 3}$. Then, when the scale $k_{0}$ is reached, it vanishes at $k=k_{0}$ as $\left(k_{0}-k\right)^{2 / 3}$. When $k$ approaches $k_{0}$, the non-linear inertial term in the hydrodynamic equation decreases and approaches the non-linear friction term. The latter becomes dominating and the cascade stops.

Let us now find how the value of $k_{0}$ in Eq.(13) is modified by the logarithmic function. The direct transfer of the energy to the heat bath $\epsilon_{\mathrm{mf}}$ is determined by the maximal vorticity:

$$
\epsilon_{\mathrm{mf}} \sim q \omega_{\max } U^{2}
$$

The total energy losses $\epsilon$ is given by

$$
\epsilon=\int_{1 / R}^{k_{0}} \frac{d k}{k} \epsilon_{\mathrm{mf}}=\epsilon_{\mathrm{mf}} \ln \left(k_{0} R\right),
$$

As a result the energy spectrum becomes

$$
E(k)=C \epsilon^{2 / 3} k^{-5 / 3} \frac{\ln ^{2 / 3} \frac{k_{0}}{k}}{\ln ^{2 / 3} k_{0} R} .
$$




\section{On developed superfluid turbulence}

From equations (31), (33) and (32) it follows that $\omega_{\max } \sim q^{1 / 2} k_{0} U \sim$ $\epsilon /\left(q U^{2} \ln \left(k_{0} R\right)\right)$. Then using the equation for energy at large scale $R, U^{2}=$ $E(k=1 / R) / R$, one obtains from Eq.(34) that the total energy losses to the heat bath is $\epsilon=U^{3} / R$. Finally one comes to the logarithmic modification of equation (13) for the scale $k_{0}$

$$
k_{0}=\frac{1}{r_{0}} \quad, \quad r_{0}=R q^{3 / 2} \ln \left(k_{0} R\right) \approx R q^{3 / 2} \ln (1 / q) .
$$

The characteristic velocity $v_{0}$ at this scale $r_{0}$ and vorticity $\omega_{\max }$ are:

$$
\omega_{\max } \sim \frac{U}{R q \ln (1 / q)}, v_{0}=\frac{\omega_{\max }}{k_{0}} \sim q^{1 / 2} U .
$$

Another model of the dispersion of the dissipation has been recently considered by Vinen in Ref. ${ }^{4}$ Vinen took into account a single term in dissipation, Eq.(19), which is peaked at large scale. He solved the Eq.(26) with this type of dissipation and obtained the solution with a stronger modification of the Kolmogorov cascade.

Here we discussed the steady state solutions of diffusion equation, but in principle, the time-dependent diffusion equation $(25)$ for $E(k, t)$ can be used for the analysis of the formation and decay of the turbulent state with different dispersion of the dissipation.

\section{Crossover to Vinen quantum turbulence}

Assuming that the superfluid turbulence is described by the nonmodified Kolmogorov cascade, let us discuss the two possible regimes which occur at different ranges of $q$. At a very small $q$ the microscopic nature of vortices with quantization of circulation becomes important. The condition of the validity of the coarse-grained hydrodynamic description used above is that the circulation relevant in the turbulent state can be considered as continuous. This means that the circulation at the smallest scale $r_{0}$ must be still larger than the circulation quantum, $v_{0} r_{0}>\kappa$ :

$$
v_{0} r_{0}=U R q^{2}=q^{2} \kappa \operatorname{Re}_{s}>\kappa, \operatorname{Re}_{s}=\frac{U R}{\kappa} .
$$

Thus the constraint for the validity of the Kolmogorov cascade is

$$
\operatorname{Re}_{s}>\frac{1}{q^{2}} \gg 1
$$

The same condition can be derived from the requirement that the characteristic scale $r_{0}$ must be much larger that the intervortex distance $l$. The latter 


\section{G.E. Volovik}

is obtained from the vortex density in the Kolmogorov state

$$
n_{\mathrm{K}}=\frac{\omega_{0}}{\kappa} \sim \frac{U}{\kappa R q}=\frac{1}{R^{2}} \frac{\operatorname{Re}_{s}}{q} .
$$

The condition $l \ll r_{0}$ leads again to the equation $v_{0} r_{0}>\kappa$ and thus to the criterion (38). Actually the same condition allows us to neglect the term in the coarse grained equation one can neglect the term which takes into account the energy of the bending of vortex lines. ${ }^{7}$

In connection to Eq.(39) let us mention the experiments on ${ }^{3} \mathrm{He}-\mathrm{B}$ in the rotating vessel. ${ }^{1}$ There the turbulent state appears as the intermediate state between the initial vortex-free state and the final state where the superfluid experiences in average the solid-body rotation $\mathbf{v}=\boldsymbol{\Omega} \times \mathbf{r}$, where $\boldsymbol{\Omega}$ is the angular velocity of the rotating vessel. In the final equilibrium state the vortex density is $n_{\text {equilibrium }}=2 \Omega / \kappa$. From Eq.(39) and taking into account that $\Omega=U / R$ one obtains that in the intermediate turbulent state the vortex density must exceed the equilibrium density:

$$
n_{\mathrm{K}} \sim \frac{1}{q} n_{\text {equilibrium }}
$$

The excess of vortices in the intermediate state has been observed in the experiment $^{1}$ and also in numerical simulations of this experiment. ${ }^{21}$

The criterion (38) contains the 'superfluid Reynolds number' $\mathrm{Re}_{s}$, which is determined by the microscopic characteristic of the vortex state - the circulation quantum $\kappa$. This superfluid Reynolds number is responsible for the crossover or transition from the classical superfluid turbulence, where the quantized vortices are locally aligned (polarized) forming thick vortex tubes (Fig. 1 top), and thus the quantization is not important, to the quantum turbulence of isolated quantized vortex lines (Fig. 1 left) whose description was developed by Vinen.

We can now consider the approach to the crossover from the quantum regime - the Vinen state which probably occurs when $\operatorname{Re}_{s} q^{2}<1$. According to Vinen ${ }^{2}$ the turbulence in the quantum regime is characterized by a single scale. The characteristic velocity is the counterflow velocity $U$, while the characteristic length scale $l$ is the distance between the vortices or the size of the characteristic vortex loops. It is determined by the circulation quantum and the counterflow velocity, $l=\lambda \kappa / U$, where $\lambda$ is the dimensionless intrinsic parameter, which probably contains $\alpha^{\prime}$ and $\alpha$. The vortex density in the Vinen state is

$$
n_{\mathrm{V}}=l^{-2} \sim \lambda^{2} \frac{U^{2}}{\kappa^{2}}=\frac{\lambda^{2}}{R^{2}} \operatorname{Re}_{s}^{2}
$$

It differs from the vortex density in the Kolmogorov state in Eq.(39) which depends not only on the counterflow velocity $U$, but also on the container 


\section{On developed superfluid turbulence}

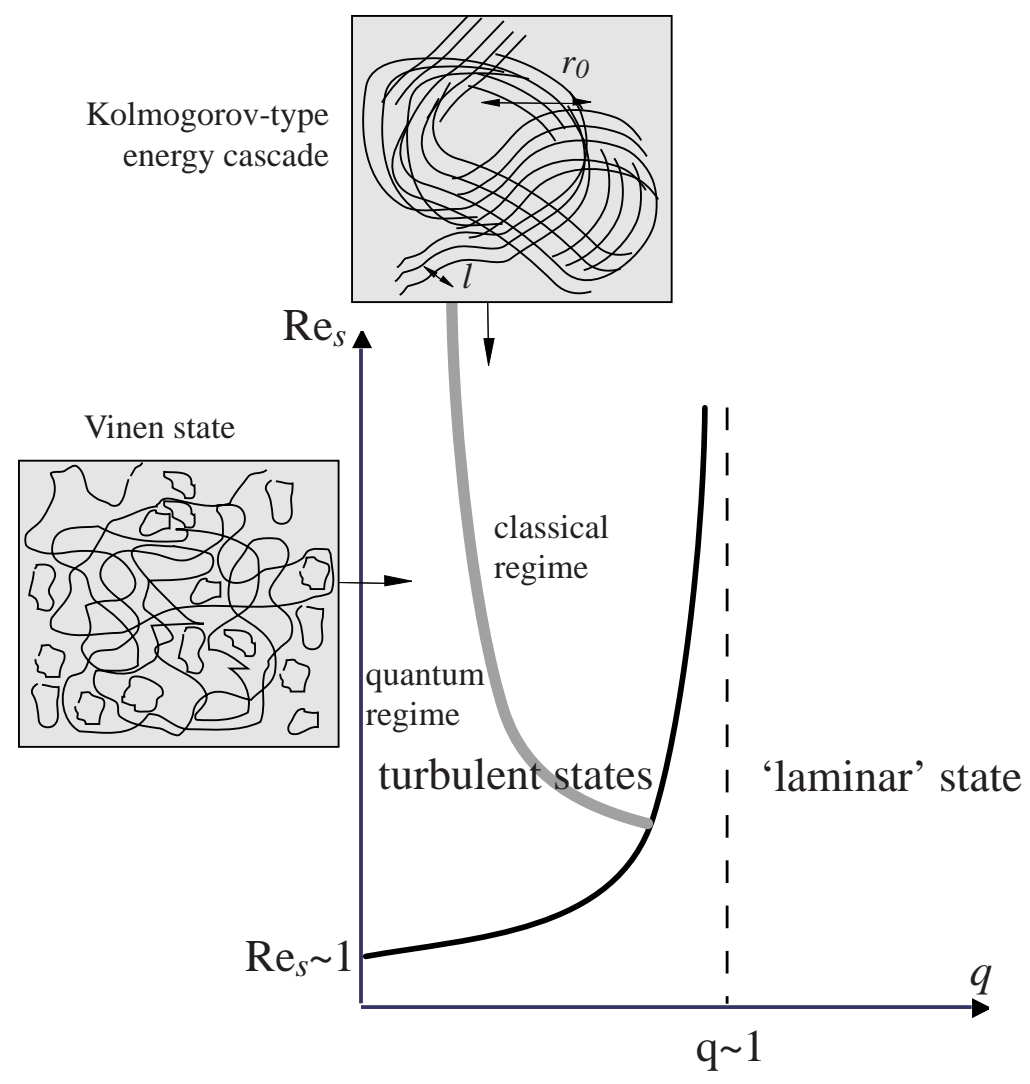

Fig. 1. Possible phase diagram of dynamical vortex states in the $\left(\operatorname{Re}_{s}, q\right)$ plane. At large flow velocity $\operatorname{Re}_{s} \gg 1$, the boundary between turbulent and 'laminar' vortex flow approaches the vertical axis $q=q_{0} \sim 1$ as suggested by experiment. ${ }^{1}$ The thick line separates two regimes of superfluid turbulence occurring at small $q$ : (i) the developed turbulence of the classical type, which is characterized by the Kolmogorov-type cascade possibly modified by the mutual friction; and (ii) the quantum turbulence of the Vinen type at even smaller $q$.

size $R$. If the crossover between the classical and quantum regimes of the turbulent states occurs at $\operatorname{Re}_{s} q^{2}=1$, the two equations (41) and (39) must match each other in the crossover region. But this occurs only if $\lambda^{2} \sim q$. If $\lambda^{2} \neq q$ there is a mismatch, and one may expect that either the two states are separated by the first-order phase transition, or there is an intermediate region where the superfluid turbulence is described by two different microscopic scales such as $r_{0}$ and $l$.

In any case the crossover between the classical and quantum regimes occurs at the border of applicability of the coarse-grained equation (3), i.e. 


\section{G.E. Volovik}

we cannot use this equation to describe the Vinen state. For this state, in which the vortex lines are not locally aligned, the Vinen equations must be used. Note that there is a principle difference between these two turbulent states, which comes from the different dependence of the vortex density on $U$ and $R$ in Eq.(41) and Eq.(39). In particular, in the Vinen state, the mutual friction force between the normal and superfluid components (the last term in Eq.(4)) obeys the cubic law suggested by Gorter and Mellink: ${ }^{23}$ $f \sim q \kappa n_{\mathrm{V}} U \sim q \lambda^{2} U^{3} / \kappa$. In the Kolmogorov state, the friction force is obeying the quadratic law, $f \sim q U \omega_{0} \sim U^{2} / R$.

Based on the above consideration one may suggest the phase diagram of different regimes of collective vortex dynamics in Fig. 1. It is possible that the boundary between the 'laminar' and 'turbulent' regions reflects the process of developing of the turbulent vortex cluster: in the 'laminar' region turbulence cannot be started by a few injected vortices, while in the turbulent region the injected vortices lead to the vortex avalance and the turbulent state. ${ }^{5}$ Actually this is what was observed in the experiment. ${ }^{1}$ However, the more fundamental role of this boundary, as the line of the phase transition between the vortex states, is not excluded: it is possible that in the laminar region the steady-state turbulence is simply unstable and decays. The instability of the turbulent vortex cluster in the lower part of the phase diagram was observed by Schwarz in his numerical simulations, but whether the boundary between the turbulent and laminar vortex flow approached the vertical axis $q=q_{0} \sim 1$ at large velocity as suggested by experiment, ${ }^{1}$ was not clear from the simulations. ${ }^{22}$

\section{Discussion}

Superfluid turbulence (as well as turbulence in classical 3-dimensional liquids) is a collective many-vortex phenomenon. Here we discussed the simple case when the normal component is at rest, or its motion is fixed (which actually occurs in ${ }^{3} \mathrm{He}-\mathrm{B}$ ). Also we considered the dynamics of a single superfluid component, i.e. we ignored the other possible superfluid components in the multi-component superfluids, such as the spin degrees of freedom in ${ }^{3} \mathrm{He}-\mathrm{B}$ : spin currents and spin vortices. But even in this simple case there can be several different types of collective dynamical vortex states. Each of these vortex states can be characterized by its own correlation functions. For example, as the characteristic which distinguishes between different vortex states one can use the behavior of the loop function

$$
g(C)=\left\langle e^{i(2 \pi / \kappa) \oint_{C} \mathbf{v} \cdot d \mathbf{r}}\right\rangle .
$$




\section{On developed superfluid turbulence}

In the limit when the length $L$ of the loop $C$ is much larger than the intervortex distance $l$ one may expect the general behavior $g(L) \sim \exp \left(-(L / l)^{\gamma}\right)$ where the exponent $\gamma$ is different for different vortex states. The asymptotic behavior of the loop function has been used for the description of the equilibrium phase transition in condensed matter and quantum field theory (see

e.g. Ref. ${ }^{24,25}$ ). It can be used also for the description of the non-equilibrium phase transition ${ }^{11}$ where vortices also appear in the intermediate state according to the Kibble-Zurek mechanism. ${ }^{26,27}$

In principle, the parameters $\alpha$ and $\alpha^{\prime}$ may depend on the type of the dynamical state, since they are obtained by averaging of the forces acting on individual vortices. The renormalization of these parameters $\alpha(L)$ and $\alpha^{\prime}(L)$, when the length scale $L$ is increasing, may also play an important role in the identification of the turbulent states, as in the case of the renormalizationgroup flow of similar parameters $\rho_{x x}$ and $\rho_{x y}$ in the quantum Hall effect (see e.g. $\left.{ }^{28}\right)$.

One can expect phase transitions between different states of collective vortex dynamics. One of such transitions, which appeared to be rather sharp, has been observed between the 'laminar' and 'turbulent' dynamics of vortices in superfluid ${ }^{3} \mathrm{He}-\mathrm{B} .{ }^{1}$ It was found that this transition was regulated by the intrinsic velocity-independent dimensionless parameter $q=\alpha /\left(1-\alpha^{\prime}\right)$, rather than by flow velocity. However, it is not excluded that both dimensionless parameters $\alpha$ and $\alpha^{\prime}$ are important. Moreover, it is also possible that only the initial stage of the formation of the turbulent state is governed by these parameters. $^{5}$

Another transition (or maybe a crossover) is suggested here between the quantum and classical regimes of developed superfluid turbulence. We argue that there is a range of parameters in the classical region of the $\left(\operatorname{Re}_{s}, q\right)$ plane where turbulence of vortex lines is described by the Kolmogorov-Richardson cascade, or by some modification of it caused by the dispersion of the dissipation. In this regime, turbulence is similar to that in classical liquids with modified dissipation. Thus the superfluid serves as a physically motivated example of the liquid with the non-canonical dissipation, which requires the general analysis of different models of dissipation and forcing (see e.g. ${ }^{17}$ ).

The turbulence in classical liquids is thought to be characterized by the dynamics of the vortex tubes, whose radii are of order of the dissipative Kolmogorov scale (see e.g. Ref. ${ }^{29}$ and references therein). In superfluid turbulence, the radii of such tubes are determined by the parameter $q$ acording to Eq.(35): $r_{0} \sim R q^{3 / 2}$. In superfluids, the classical description is valid when these vortex tubes are fat enough, i.e. they contain many quantized vortices. The circulation around the tubes essentially exceeds the circulation quantum $\kappa$, so that we can ignore the quantum nature of superfluid vortices. In 


\section{G.E. Volovik}

this region we can study analytically, numerically and experimentally all the phenomena which take place in classical liquids, including intermittency.

The crossover to the quantum regime occurs when the circulation around the relevant vortex tubes becomes comparable to the circulation quantum $\kappa$. According to Vinen, in the quantum regime, turbulence is represented by a single length scale - the intervortex distance $l$ - and the single velocity scale $U$, which are related through the circulation quantum: $l \sim \kappa / U$. It was suggested by Skrbek ${ }^{30}$ that such a crossover between the quantum Vinen state and the classical Kolmogorov state of superfluid turbulence was probably observed in several experiments with the counterflowing superfluid ${ }^{4}$ He. Skrbek identified these states with turbulent states I and II according to the Tough's classification scheme. ${ }^{31}$

\section{ACKNOWLEDGMENTS}

I thank V.B. Eltsov, D. Kivotides, N.B. Kopnin, M. Krusius, V.V. Lebedev, L. Skrbek, F. Toschi, M. Tsubota and W.F. Vinen for fruitful discussions and criticism. This work was supported by ESF COSLAB Programme and by the Russian Foundation for Fundamental Research.

\section{REFERENCES}

1. A.P. Finne, T. Araki, R. Blaauwgeers, V.B. Eltsov, N.B. Kopnin, M. Krusius, L. Skrbek, M. Tsubota, and G.E. Volovik, An intrinsic velocity-independent criterion for superfluid turbulence, Nature 424, 1022-1025 (2003).

2. W.F. Vinen, Proc. R. Soc.. London, ser A 242, 493 (1957).

3. W.F. Vinen, and J.J. Niemela, J. Low Temp. Phys. 128, 167 (2002).

4. W.F. Vinen, Superfluid turbulence in the presence of a stationary normal fluid, to be published in Phys. Rev. B

5. N.B. Kopnin, Vortex instability and the onset of superfluid turbulence, Phys. Rev. Lett. to be published; cond-mat/0309708.

6. G.E. Volovik, 'Classical and quantum regimes of the superfluid turbulence', Pisma ZhETF 78, 1021-1025 (2003) [JETP Letters 78, 533-537 (2003)].

7. H.E. Hall, in: Liquid Helium, International School of Physics "Enrico Fermi" Course XXI, G. Carreri, ed. , Academic Press, New York (1963); Phil. Mag. Supplement 9, issue 33, 89 (1960).

8. E.B. Sonin, Rev. Mod. Phys. 59, 87 (1987).

9. N.B. Kopnin, Theory of Nonequilibrium Superconductivity, Clarendon Press, Oxford (2001).

10. T.D.C. Bevan et al., Nature, 386, 689 (1997); J. Low Temp. Phys, 109, 423 (1997).

11. G.E. Volovik, The Universe in a Helium Droplet, Clarendon Press, Oxford (2003). 


\section{On developed superfluid turbulence}

12. W.D. McComb, The Physics of Fluid Turbulence, Clarendon Press, Oxford (1990).

13. K.W. Schwarz, Fluid dynamics of a quantized vortex filament in a hole, Physica B 197, 324 (1994); Numerical experiments on single quantized vortices, preprint.

14. W. F. Vinen, Makoto Tsubota, and Akira Mitani, Kelvin-wave cascade on a vortex in superfluid ${ }^{4} \mathrm{He}$ at a very low temperature, Phys. Rev. Lett. 91, 135301 (2003).

15. Makoto Tsubota, Tsunehiko Araki and Sergey K. Nemirovskii, Dynamics of vortex tangle without mutual friction in superfluid ${ }^{4} \mathrm{He}$, Phys. Rev. B 62 1175111762 (2000).

16. Thibault Damour and Alexander Vilenkin, Gravitational wave bursts from cusps and kinks on cosmic strings, Phys.Rev. D 64, 064008 (2001).

17. L. Biferale, M. Cencini, A. S. Lanotte, M. Sbragaglia and F. Toschi, Anomalous scaling and universality in hydrodynamic systems with power-law forcing, New J. Phys. 6, 37 (2004) .

18. C. Leith, Phys. Fluids 10, 1409 (1967); Phys. Fluids 11, 1612 (1968).

19. Colm Connaughton and Sergey Nazarenko, Warm Cascades and Anomalous Scaling in a Diffusion Model of Turbulence, Phys. Rev. Lett. 92, 044501 (2004).

20. Makoto Tsubota, Kenichi Kasamatsu, Tsunehiko Araki, Dynamics of quantized vortices in superfluid helium and rotating Bose-Einstein condensates, condmat/0309364.

21. Makoto Tsubota, private communications.

22. W.F. Vinen, private communications.

23. C.J. Gorter and J.H. Mellink, On the irreversible processes in liquid helium II, Physica 15, 285-304 (1949).

24. G. Toulouse, Gauge concepts in condensed matter physics, in: Recent Developments In Gauge Theories, 331-362 (1979).

25. A.M. Polyakov Gauge Fields and Strings, Contemporary Concepts in Physics 3, Harwood Academic, Chur (1987).

26. T.W.B. Kibble, Topology of cosmic domains and strings, J. Phys. A 9, 13871398 (1976).

27. W.H. Zurek, Cosmological experiments in superfluid helium?, Nature 317, 505 (1985).

28. D.E. Khmelnitskii, Quantization of Hall conductivity, Pis'ma ZhETF 38, 454458 (1983).

29. J.-P. Laval, B. Dubrulle, S. Nazarenko, Non-locality and Intermittency in 3D Turbulence, Phys. of Fluids 13, 1995-2012 (2001).

30. L. Skrbek, Flow phase diagram for the helium superfluids, cond-mat/0402301.

31. J.T. Tough, Superfluid turbulence, in: Prog. in Low Temp. Phys., vol. VIII, North Holland, Amsterdam (1982). 